\title{
La construcción de un lector democrático ANUAL DA de masas. Borges en el diario Crítica de Buenos Aires y la Historia Universal de la Infamia
} CONFERÊNCIA

\author{
The construction of a democratic reader of the masses. Borges on the newspaper \\ Crítica and on the "Historia Universal de Ia Infamia"
}

\section{A construção de um leitor democrático de massas. Borges no jornal Crítica de Buenos Aires e na Historia Universal de Ia Infamia}

\section{Pablo Calvi}

Profesor asistente en la Escuela de Periodismo de Stony Brook University e Director Asociado para América Latina del Marie Colvin Center for International Reporting. Ph.D. por la Escuela de la Universidad de Columbia de Periodismo en el año 2011. <pablo.calvi@stonybrook.edu>

\section{RESUMEM}

El objetivo de este trabajo es analizar HUI y, en particular, una de las historias más destacadas de la serie"El proveedor de iniquidades Monk Eastman", bajo el contexto definido por las formas de producción de un diario tabloide como Crítica, pero además comprender este trabajo como una lectura destinada al entretenimiento sabatino de los lectores de clase media baja que, a principios de 1930, leían Crítica en Buenos Aires. Para ello intentaremos convertirnos en "tenebrosos cisnes" usando la imagen que Borges forjó en el prólogo a su edición 1935 de la HUI para definir a sus lectores ideales. Nuestro objetivo final para este trabajo es revelar algunos mecanismos aún no explorados en el estudio de estos textos fundacionales, mecanismos que nos ayuden a comprender el posicionamiento de Borges frente la prensa diaria en la Argentina de la década de 1930.

\section{ABSTRACT}

The article analyzes the "Historia Universal de la Infamia" (HUI), particularly one of the most prominent stories of the series "El provedor de iniquidades Monk Eastman", within the context defined by the modes of production of a tabloid newspaper such as Crítica, from Buenos Aires. In order to achieve this goal, we have attempted to become ourselves "dark swans" - to use Borges' image of the perfect reader in the prologue of the 1935 edition of HUI. The main objective is to reveal some of the mechanisms that have not yet been exploited in former analysis of such texts, mechanisms that help us to understand Borges' approach towards the Argentine daily press of the 1930s.

Keywords: Journalism. Democracy. Jorge Luis Borges.

\section{RESUMO}

O objeto deste trabalho é analisar a "Historia Universal de la Infamia" (HUI) e, particularmente, uma das histórias mais sobressalientes da série "El provedor de iniquidades Monk Eastman", sob o contexto definido pelas formas de produção de um jornal tabloide como Crítica, em Buenos Aires. Para isso, tentamos tornarnos "tenebrosos cisnes", usando a imagem proposta por Borges no prólogo da sua edição de 1935, da HUI, para definir os seus leitores ideais. Nosso objetivo final neste trabalho é revelar alguns mecanismos ainda não explorados no estudo destes textos fundacionais, mecanismos que nos ajudem para compreender o posicionamento de Borges diante à imprensa diária na Argentina da década de 1930.

Palavras-chave: Jornalismo. Democracia. Jorge Luis Borges. 
Desde que comienza a ser publicada, la obra de ficción de Jorge Luis Borges recibió la atención obsesiva de expertos y académicos en todo el mundo. La bibliografía secundaria que estos trabajos han desencadenado puede, por sí misma, llenar bibliotecas enteras. Pero los ejercicios tempranos de la narrativa Borgeana, algunos trabajos que podrían clasificarse de un modo amplio como no ficción (volveremos a esto más adelante), sólo recientemente han empezado a despertar el interés de la crítica. La Historia Universal de la Infamia (HUI), el primer esfuerzo narrativo del escritor argentino, se compone de una serie de cuentos publicados por entregas entre el 12 de agosto de 1933 y el 20 de enero de 1934 en la Revista Multicolor de los Sábados, una sección del desaparecido vespertino porteño Crítica. Las historias que Borges agrega en esta serie fueron publicadas en un único volumen en 1935 y luego revisadas y reeditadas por Borges en 1954.

La relevancia de los textos de HUI en la historia de la narrativa (de ficción y periodística) en América Latina es polivalente. Por un lado, como ya ha sido señalado por la mayoría de los críticos que estudiaron esta zona discreta de la obra de Borges, estos relatos muestran el trabajo de formación de la prosa de un autor que llegará a convertirse en uno de los mayores exponentes de la ficción del siglo 20. Por otro lado -y para nosotros esto es central - HUl abre una ventana al interior de las nociones borgeanas de objetividad y propone una mirada ante la idea de Borges sobre el papel de la prensa de masas en la joven democracia Argentina de principios del siglo 20. Por último, los recuerdos del propio Borges acerca del tiempo en el cual estos textos fueron producidos, permiten apreciar su trabajo como periodista, narrador de hechos verídicos, y editor de una revista de entretenimientos sabatina, durante una década que ha sido bautizada por el periodista argentino José Luis Torres como "Década Infame" debido a los innumerables actos de corrupción gubernamental, fraude electoral y crimen político que tuvieron lugar entonces. La conexión que se puede establecer entre la llamada "Década Infame", la serie de textos aparecidos en el suplemento de Crítica y la Historia Universal de la Infamia es, cuanto menos, fascinante. Pero todos estos elementos adquieren una relevancia aún mayor si se considera que Borges fue uno de los escritores más innovadores del siglo 20.

El objetivo de este trabajo es analizar HUI y, en particular, una de las historias más destacadas de la serie "El proveedor de iniquidades Monk Eastman", bajo el contexto definido por las formas de producción de un diario tabloide como Crítica, pero además comprender este trabajo como una lectura destinada al entretenimiento sabatino delos lectores de clase media baja que, a principios de 1930 leían Crítica en Buenos Aires. Para ello intentaremos convertirnos en "tenebrosos cisnes" usando la imagen 
que Borges forjó en el prólogo a su edición 1935 de la HUI para definir a sus lectores ideales. Nuestro objetivo final para este trabajo es revelar algunos mecanismos aún no explorados en el estudio de estos textos fundacionales, mecanismos que nos ayuden a comprender el posicionamiento de Borges frente la prensa diaria en la Argentina de la década de 1930.

\section{Historia universal de la infamia, su impacto y relevancia}

Los relatos a los que Jorge Luis Borges Ilamó Historia universal de la infamia aparecieron entre el 12 de agosto de 1933 y el 20 de enero de 1934 como serie, dentro de un suplemento de entretenimiento para el fin de semana publicado por el diario Crítica de Buenos Aires: la Revista Multicolor de los Sábados. HUI fue, en palabras de Borges, "el verdadero comienzo de mi vida como escritor". Precursora entre las publicaciones de entretenimiento, La Revista Multicolor abundaba en lecturas livianas, extensas, secciones de humor y juegos. Pero sería imposible comprender su impacto y dimensión sin antes analizar algunos aspectos de Crítica, el periódico dentro del cual aparecía la Revista Multicolor.

Crítica era un diario especial. Vespertino de formato sábana, creado en 1913 por el periodista uruguayo Natalio Botana, Crítica instaló un contrato de lectura absolutamente novedoso para la prensa rioplatense de principios de siglo, basado en la complicidad dialógica entre periodistas y lectores, un estilo en parte heredado de las revistas populares de la década de 1920 en Buenos Aires (Calvi, 2011). Pero Crítica apelaba a un público mucho más sofisticado que el de la prensa política argentina de fines del siglo 19. El diario asumía la misión de informar a sus lectores, pero también se proponía conectarse con ellos emocionalmente, y sobre todo entretenerlos. Siguiendo el estilo de la "Prensa del Centavo" estadounidense, Crítica utilizaba titulares de cuerpo grande, ilustraciones y fotos en tapa, estas últimas muchas veces tomadas por los propios lectores (Saitta, 1998, p. 88).

Gracias a la enorme popularidad que adquirió a poco de su lanzamiento entre las clases medias y trabajadoras, Crítica comenzó a autoproclamarse "la voz del pueblo" (Saítta, ${ }^{1998)}$. Su estilo y sus noticias, que apelaban a los trabajadores de las flamantes oficinas comerciales de Buenos Aires - el sector más dinámico de una clase media en auge - tuvieron un impacto en la prensa Argentina y latinoamericana equivalente al que el New York World de Joseph Pulitzer y el New York Journal de William Randolph Hearst tuvieron en la prensa estadounidense casi cien años antes.

En Crítica, Botana introdujo las técnicas de la novela serial a la redacción de noticias en la Argentina, y agregó la noción de efecto dramático a la escritura 
periodística en un tono similar a lo que hoy se conoce como "periodismo amarillo". Sin embargo, el elemento más innovador que Botana incorpora al periodismo latinoamericano es la idea de que las noticias deben también tener una "voz literaria". Para ello, a principios de 1930, Botana contrató a un grupo de escritores de ficción y poetas profesionales ya establecidos para elevar la calidad de la prosa de Crítica y, en última instancia, del periodismo de redacción. A raíz de estos cambios, Crítica comenzó a publicar en 1933 la Revista Multicolor de los Sábados a cargo de Jorge Luis Borges y el poeta Ulises Petit de Murat.

La literatura académica sobre Crítica, la Revista Multicolor de los Sábados y HUI, no es abundante, aunque ha comenzado recientemente a acumularse. Sin embargo la bibliografía sobre estos tres objetos es desigual, pues es en particular la obra de Borges la que ha recibido mayor atención.

En cuanto a los estudios académicos sobre Crítica y su impacto en el ecosistema de medios en Argentina y América Latina, Regueros de Tinta (1998), de Sylvia Saítta, ofrece un análisis histórico de suma importancia. En su estudio, la crítica revela en qué medida Botana utilizaba el vespertino como engranaje de poder político. Crítica legitimó su influencia en el discurso público mediante la cuantificación de su importancia (en cantidad de lectores diarios) haciendo público de un modo permanente el crecimiento en su tirada, el número de sus ediciones y la llegada al público, argumenta Saítta (1998). Gracias a su influencia sobre el gran público, Botana se convirtió en pocos años en un actor político temido y adulado por gobernadores y presidentes. Para consolidarse como el poderoso líder de opinión que llegó a ser, Botana prestaba particular atención a las acciones de caridad: Crítica donó "Cien mil juguetes a los niños necesitados"; "Crítica otorgará una Feliz Navidad a los parados. Hemos recogido una donación de 400 kilos de pan dulce para ellos "(Saitta, 1998, p. 138-154). La consolidación de su influencia política le permitió a Botana expandir aún más su emporio de medios de comunicación con una estación de radio y corresponsales en las provincias y en el extranjero. En los años posteriores a 1923, Crítica, se convirtió en un actor social central que satisfacía las crecientes demandas culturales y políticas de sus lectores (Saítta, 1998, p. 129-130). Tenía una tirada de 166.385 en sus tres ediciones de la tarde, y llegó a ser el tercer periódico de mayor tirada en la Argentina después de La Prensa y La Nación - este último con una tirada de 188.835. En 1927 Crítica incorporó a su redacción una sección llamada "Atención al Público" a la que se le asignaba un reportero rotativo que asumía el puesto como una forma leve de castigo (Saítta, 1998, p. 130-131). El periodista a cargo tendía a una "caravana de suplicantes" que concurría diariamente a exponer sus conflictos y penurias a la sala de redacción. La sección era un "Muro de los 
Lamentos", un lugar en el que "el redactor tiene que demostrar una paciencia digna de Job y estoica resignación para escuchar súplicas y lamentaciones, contrariedades y desgracias" (Talice, 1989, p. 492). Esta sección, informalmente conocida como "la sección de la miseria" tenía un fondo de caja de hasta 10 pesos, que se utilizaba de forma discrecional para atender las necesidades más urgentes de algunos de los lectores" (Saítta, 1998, p. 131).

Pero Crítica no se consolidó solamente en base a sus acciones de caridad. Paradójicamente, en la búsqueda de relevancia política, el vespertino debió irrumpir en el campo de la cultura, principalmente a través de la Revista Multicolor. Debemos el estudio inaugural del suplemento al historiador y crítico de medios argentino Jorge B. Rivera. En “Los Juegos de un tímido: Borges en el suplemento de Crítica", Rivera describe la conexión entre la vanguardia literaria argentina de la década de 1920 y el nuevo periodismo que Botana promovía desde las páginas de su diario. La relación con el público de masas ayudó a escritores poco conocidos a acceder al imaginario de las audiencias masivas en expansión.

En su estudio, Rivera asegura que para Botana había un tipo de literatura vital, real, que el público debía conocer directamente y no por terceros. Siguiendo esa idea, el editor aprovechó la enorme circulación de su periódico para volver accesible series de textos que no estaban inmediatamente al alcance de los sectores populares. Rivera ofrece como ejemplo las ediciones Crítica de libros populares (Rivera, 1976, p. 22).

Ya en 1930, Botana vio la oportunidad de contratar a un colectivo de escritores entre los que se encontraban los más innovadores en América Latina, para cubrir las secciones de arte, literatura, e incluso la sección policial. Esta jugada tuvo como resultado una revolución literaria y periodística en Buenos Aires que pronto se extendió por todo el continente (Rivera, 1976, p. 22).

Como se mencionó anteriormente, de los tres ángulos posibles para estudiar el trabajo de Borges en la Revista multicolor de Crítica, ha sido la producción misma de Borges para el suplemento la que ha recibido la mayor atención por parte de los críticos. Inmediatamente exitosos y elogiados de forma ubicua, los cuentos de Borges para la Revista Multicolor fueron altamente considerados por la crítica desde su publicación. "Borges narrador" del crítico Amado Alonso, uno de los primeros estudios sobre la prosa de Borges, fue publicado casi de inmediato, en 1935, en la legendaria revista literaria Sur dirigida por Victoria Ocampo. El mecanismo fundamental que subyace a la HUI, el oxímoron, fue minuciosamente estudiado por Jaime Alazraki en Oxymoronic Structure in Borges Essays. Alazraki produjo varios artículos dedicados a sondear en las innovaciones literarias introducidas por Borges en HUI. Daniel Balderston 
exploró la relación antagónica de Borges con el realismo social de América Latina en Out of Context: Historical Reference and the Representation of Reality in Borges, y hace un seguimiento en profundidad de "Monk Eastman, Proveedor de iniquidades" en su ensayo "Borges and the Gangs of New York". Sin embargo, el estudio más exhaustivo, completo y detallado del trabajo de Borges para la Revista Multicolores fue el libro de Raquel Atena Green Borges y Revista Multicolor de los Sábados, una revisión y análisis de cada uno de los relatos originales que Borges publica en Crítica. En este estudio, Green analiza tanto las piezas que Borges firmó y publicó más tarde como libro, como las que permanecieron apócrifas y, durante algún tiempo, estuvieron perdidas u olvidadas. En este trabajo Green describe con detalle las fuentes de Borges y sus referencias intertextuales, apoyándose en documentos personales, largas entrevistas y conversaciones con el mismo Borges.

De estos tres diferentes tipos de estudios, los que se enfocan en Crítica generalmente discuten las condiciones históricas y culturales que hicieron posible la aparición de la prensa popular en Argentina, y su papel en relación con los nuevos lectores de masas. Los estudios sobre la Revista Multicolor suelen abordar las particularidades del suplemento, sus plumas y las conexiones de la revista con la vanguardia literaria que tuvo lugar en la Argentina de la década de 1920. Finalmente, los estudios que se centran en HUI abordan los mecanismos literarios que Borges utiliza para construir sus historias, las fuentes de estos relatos y sus referencias históricas, y estudian de manera especial las falsas atribuciones, un mecanismo que se convirtió en un elemento diferencial del estilo narrativo de Borges.

Hay, sin embargo, en esta bibliografía, un punto ciego que intentaré reclamar en este ensayo: la conexión entre los primeros relatos de Borges, los temas de no ficción escritos para los lectores de Crítica, y el contexto político de la década de 1930 en Buenos Aires. En el estudio de esta tríada, propongo explorar el tipo de lector que Borges estaba ansioso por cultivar en la prensa popular, y el tipo de actividad de lectura que él mismo alentó basada en un sistema de escritura particular anclado en la antífrasis. Por último, este estudio conectará a Borges con un género al que aún no se lo ha asociado: el "embuste" (conocido en inglés como the hoax).

Con el fin de explorar esta conexión, deberemos en primer lugar tomar un pequeño desvío. 


\section{Borges y las bandas de Buenos Aires}

En su ensayo de 2003 Borges and the Gangs of New York, Daniel Balderston presta especial atención a la segunda historia que Borges publicó en la Revista Multicolor de los Sábados, "El proveedor de iniquidades Monk Eastman". Balderston señala correctamente que el cuento de Borges sigue de cerca The Gangs of New York, un libro de cariz histórico escrito por el estadounidense Herbert Asbury y publicado en 1928. La clave es que, como los críticos de Borges ya saben esperar, muchas de las atribuciones que utiliza el escritor argentino son engañosas y por momentos completamente fabricadas'.

Balderston (2003) identifica tres diferencias fundamentales que separan la historia de Monk Eastman escrita por Borges del original. Eastman era un gángster de origen judío, nacido en Williamsburg. Pero de acuerdo con el ensayo de Balderston, el retrato de Borges presenta además un número limitado de datos que han sido alterados para amplificar la textura cinemática de la narración. A continuación, cito Balderston (2003) en detalle, incluyendo sus referencias de Borges, para simplificar el análisis:

In "El proveedor de iniquidades" the added details have largely to do with Eastman's relations to his cats and pigeons, a detail mentioned by Asbury. In other words, the grand epic scenes the battle of Rivington, for instance - are closely based on the Asbury book, but Borges adds a few telling details to make the story more vivid. An example of this technique is the mention of the dead after the "battle of Rivington" (Balderston, 2003). Asbury (1928) writes: It was not until the reserves from several stations charged down Rivington street with roaring revolvers that the thugs left their dens. They left three dead and seven wounded upon the field, and a score were arrested before they could get away. Borges ends the section on this incident: Debajo de los grandes arcos de ingeniería quedaron siete heridos de gravedad, cuatro cadáveres y una paloma muerta. The addition of the dead pigeon is a reminder of Eastman's tenderness with his pets, who accompanied him everywhere; it is as sign of Borges's fascination with the cinematic detail at the time of this writing.

A similar example is that of Eastman's death. Asbury (1928) writes: On May 3, 1919, Governor Smith signed an executive order

1 En un artículo anterior me he referido al posible origen de las citas y atribuciones erróneas de Borges, de sus distorsiones y exageraciones, en la literatura sarmientina (Calvi, 2011). Pero, como ya ha sugerido Molloy (2000, p. 19-47), estas desviaciones en la narrativas borgeana basada en hechos están además relacionadas con su deseo de dinamitar la naturaleza fija de texto, crear inestabilidad y fomentar la creatividad y la imaginación en lectores. En este orden de ideas, la conexión de Borges con el realismo mágico parece, al menos, indirecta, si no inexistente. 
restoring Monk Eastman to full citizenship, and the former king of the gangsters said that he would go straight. The police obtained a job for him, and he did not again come to their attention until the morning of December 26, 1920, when his body was found lying on the sidewalk in front of the Blue Bird Café at No. 62 East Fourteenth street, near Fourth avenue. He had been shot five times and was dead. A few days later Eastman was buried with full military honors, and in December, 1921, Jerry Bohan, a Prohibition Enforcement Agent, pleaded guilty to manslaughter in the first degree and was sentenced to prison for from three to ten years. He was paroled late in 1923. Bohan said that he had quarrelled with Eastman over tipping a waiter, but when detectives began to investigate they found that Monk had been bootlegging and selling dope. Borges's final section is much more succinct, and bears the title "El misterioso, lógico fin": El 25 de diciembre de 1920 el cuerpo de Monk Eastman amaneció en una de las calles centrales de Nueva York. Había recibido cinco balazos. Desconocedor feliz de la muerte, un gato de lo más ordinario lo rondaba con cierta perplejidad. Once again the detail of Eastman's pet, which had accompanied him even on his final mission, replaces all the information from the police blotter except for the date (changed to one day earlier to coincide with Christmas) and the five fatal shots (Balderston, 2003, p. 28-30).

Como muestra un análisis textual en proximidad, la lectura de Balderston hace dos omisiones importantes: en primer lugar, su ensayo evita mencionar el primer párrafo del cuento de Borges, un texto que funciona como marco narrativo para el relato. Este párrafo de 104 palabras en el original español (121 palabras en su traducción al inglés), aparece en original del suplemento de Crítica, el 19 de agosto de 1933, sin el subtítulo que luego Borges le añadirá a la primera edición del libro en 1935: "Los de esta América”. Balderston, de este modo, deja fuera de su estudio una sección inaugural clave con la que Borges en marca su relato ante el lector local y -como sostengo aquí- traza las coordenadas para la construcción de un contexto histórico.

El análisis de Balderston incurre en una segunda omisión, la de algunos elementos contextuales que rodearon la publicación de "Monk Eastman..." en la edición sabatina del diario Crítica, elementos sumamente visibles para los lectores original es del relato y centrales para una nueva lectura del texto. Es posible argumentar que estas dos omisiones son clave para comprender la relación de Borges con el periodismo de principios del siglo 20; revelan un énfasis en la utilización de ciertos recursos literarios que no ha sido abordado hasta ahora, y ayudan a entender algunas de las ideas de Borges en relación con 
la realidad y la ficción en el contexto de la democracia incipiente en la Argentina. Nos detendremos en ellos en la siguiente sección.

Finalmente junto con una larga lista de críticos Alonso (1935), Alazraki (1971) Balderston (1993) ha sugerido que la figura retórica fundacional de HUI es el oxímoron. Historia universal de la infamia, que es el título de la serie; “El Cruel Redentor Lázarus Morell", la primera pieza narrativa firmada por Borges, publicada el 12 de agosto de 1933; "Monk Eastman, Proveedor de iniquidades", el segundo relato publicado el 19 de agosto de 1933; y la mayoría de las historias en la HUI recurren a este dispositivo que yuxtapone términos de valor semántico contradictorio. Pero existe otro mecanismo en funcionamiento que sólo puede encontrarse cuando se opera un alejamiento hacia afuera del análisis textual y se comienza a considerar algunos de los textos con los que HUI convivió en Crítica, en el mes de agosto de 1933.

Por último, escasamente se ha mencionado en estudios previos la tradición hagiográfica que influye de modo notable en HUI. Las "vidas de santos", como género, tienen una larga historia en la literatura latinoamericana, y las historias de antihéroes y anti-santos fueron una constante en la construcción del periodismo literario moderno en América Latina, comenzando con Facundo el extenso ensayo - perfil, estudio sociológico, novela de folletín-sobre Facundo Quiroga que Domingo Sarmiento publica en 1845. Históricamente, más próximas a Borges, hay varias hagiografías anti-heroicas fundamentales escritas por José Martí, publicadas por La Opinión Nacional de Caracas y republicadas por La Nación de Buenos Aires entre fines del 1800 y principios de 1900 , relatos que difícilmente Borges haya ignorado. Aunque no hay referencias directas en la bibliografía que estudia a Borges o en las entrevistas al escritor, es interesante observar que "El asesino desinteresado Bill Harrigan", presenta una sorprendente semejanza estructural y estética con el texto martiano "Jesse James, Gran bandido " - vale además notar el uso del oxímoron en este título de Martí - un obituario de Jesse James que el cubano escribió para La Opinión Nacional de Caracas en $1882^{2}$.

2 A modo de ejemplo, copio a continuación los párrafos introductorios de Martí en su relato sobre Jesse James, primero, seguido del texto de Borges sobre Billy The Kid. Obsérvese el uso de descripción metafórica y el andamiaje oximorónico en ambos textos. "Estos días que para Nueva York fueron de fiesta, han sido de agitación grande en Missouri, donde había un bandido de frente alta, hermoso rostro y mano hecha a matar, que no robaba bolsas sino bancos; ni casas sino pueblos; ni asaltaba balcones sino trenes. Era héroe de la selva. Su bravura era tan grande, que las gentes de su tierra se la estimaban por sobre sus crímenes. Y no nació de padre ruin, sino de clérigo, ni parecía villano, sino caballero, ni casó con mala mujer, sino con maestra de escuela. Y hay quien dice que fue cacique político, en una de sus estaciones de reposo, o que vivía amparado de nombre falso, y vino como cacique a elegir Presidente a la última convención de 
Pero para comprender la importancia de estos elementos en HUI es necesario tener en cuenta algunos procesos históricos como parte del contexto en el que Borges escribió sus relatos.

\section{Algunos elementos contextuales}

Tras la crisis económica de 1890, Buenos Aires (Capital Federal de la República Argentina desde 1880) entró en su primera fase de suburbanización. Oleadas de inmigrantes internos y extranjeros atraídos por la comodidad de una metrópoli moderna, dispararon un importante crecimiento poblacional en la capital. Buenos Aires pronto se convirtió en una ciudad cosmopolita: calles pavimentadas con adoquines; un sistema de alcantarillado eficiente; la primera red eléctrica de América Latina; un sistema de limpieza urbana meticuloso; una red de seguridad social con hospitales y clínicas de clase mundial; la red de transporte público con tranvías tirados por caballos en 1870, y a partir de 1898 movidos por electricidad; y el primer sistema de metro en América Latina en 1913 hicieron de Buenos Aires un lugar deseable para propios y extraños.

A principios de 1900, Argentina comienza a consolidar un joven sistema democrático. Gracias a la aprobación en 1912 de la Ley Sáenz Peña - que establecía el sufragio masculino secreto, universal y obligatorio - en 1916 Hipólito Yrigoyen se consagró como en el primer presidente argentino refrendado popularmente. La Ley Nacional de Educación - Ley Láinez - aprobada el 26 de junio de 1884, otorgaba el derecho a la educación primaria gratuita y laica a todos los residentes en suelo argentino. Ese proyecto de ley también jugó un papel decisivo en la profesionalización de los trabajadores intelectuales, y tuvo un impacto central en la pronunciada caída de las tasas de analfabetismo durante las siguientes décadas - de un $78 \%$ en 1869, al 35\% en 1914. Pero las altas tasas de alfabetización tuvieron además un resultado paralelo: el desarrollo de un creciente público lector (Eujanian, 1999, p. 21).

los demócratas. Están las tierras de Missouri y las de Kansas llenas de recio monte y de cerradas arboledas. Jesse James y los suyos conocían los recodos de la selva, los escondrijos de los caminos, los vados de los pantanos, los árboles huecos. Su casa era armería, y su cinto otra, porque llevaba a la cintura dos grandes fajas, cargadas de revólveres. Empezó a vivir cuando había guerra, y arrancó la vida a mucho hombre barbado, cuando el aun no tenía barba" (Martí, 2000, p. 38-41). "La imagen de las tierras de Arizona, antes que ninguna otra imagen: la imagen de las tierras de Arizona y de Nuevo Méjico, tierras con un ilustre fundamento de oro y plata, tierras vertiginosas y aéreas, tierras de la meseta monumental y de los delicados colores, tierras con blanco resplandor de esqueleto pelado por los pájaros. En esas tierras otra imagen, la de Billy the Kid: el jinete clavado sobre el caballo, el joven de los duros pistoletazos que aturden el desierto, el emisor de balas invisibles que matan a distancia, como una magia. El desierto veteado de metales, árido y reluciente. El casi niño que al morir a los veintiún años debía a la justicia de los hombres veintiuna muertes - sin contar mejicanos " (Borges, 1999, p. 65). 
El mercado de lectura argentino se desarrolló de un modo particular. Con conocimientos técnicos de lectoescritura, pero sin los hábitos o el interés para navegar un catálogo de libros o una biblioteca pública, los nuevos lectores urbanos, deseosos de participar en un entorno cambiante y cada vez más sofisticado, se hallaron en la necesidad de comprar periódicos y revistas directamente disponibles en quioscos.

En los años 1930s, desde sus talleres en Avenida de Mayo 1333, el diario fundado por Natalio Botana era entonces el segundo vespertino de mayor circulación en la Argentina con un promedio de 300 mil ejemplares diarios (Saítta, 1998, p. 82; 246). Por aquellos días, sus cinco ediciones de entre 20 y 40 páginas, comenzaban a configurar un pacto de lectura alejado del de la prensa partidaria, un nuevo modelo en el que, según Saítta,

lejos ha quedado la caracterización del pueblo como la 'plebe iletrada'... En los años veinte, Crítica inaugura un estilo de interpelación y reconfigura el modo en que se presenta a sus lectores: Crítica se instituye a sí mismo como "la voz" de la masa ciudadana transformando el éxito de mercado en criterio de legitimación (Saítta, 1998, p. 61).

La puesta en valor del mercado como vértice de legitimación política, social y simbólica funciona en varios planos. Por un lado, a diferencia de la prensa de partido (el modelo de prensa que la precede a la prensa masiva y popular), Crítica interpela a sus lectores como pares en una relación de iguales. El acceso al capital simbólico en la Argentina de los años 1930s comenzaba a definirse no ya directamente en función de la pertenencia de clase, sino a partir del acceso al sistema de educación pública y a los medios de información. Y en parte gracias a Crítica, y en gran medida gracias a otras publicaciones de la época, la información política, social y cultural más destacada comienza a volverse accesible a grupos amplios de trabajadores urbanos de clases media y baja.

La consolidación democrática y el aumento en los niveles de alfabetismo tras la sanción de la ley Láinez trajeron aparejado además un crecimiento vertiginoso del lectorado, que se volcaría al consumo del periodismo urbano en una profusión de estilos y formatos ${ }^{3}$. En este sentido, la reducción del precio

3 En 1906, gracias a la Ley Láinez, el gobierno argentino destina 10 millones de pesos a la creación de 700 nuevas escuelas. Y a pesar de las altas tasas de deserción (algunas veces por encima del 75\%), en 1910 la mayor parte de la población en edad escolar en la Argentina ya sabía leer y escribir (Eujanián, 1999, p. 21). 
de tapa - una estrategia comercial que Crítica comienza a utilizar en los años 1920s y mantiene inalterada casi hasta su cierre - reproduce en la Argentina un modelo de prensa barata popularizado casi un siglo atrás en los Estados Unidos por los empresarios periodísticos William Randolph Hearst y Joseph Pulitzer.

\title{
Hacia un periodismo de masas: 1920-1930
}

En la Argentina de fines de los años 1920s y principios de los 1930s el público de masas que comienza a consolidarse junto con el crecimiento de la ciudad y los mercados urbanos ha dejado de funcionar como un recipiente vacío que debe ser colmado de información. Las "tribunas de doctrina" de la tradicional prensa de elite se han vuelto anacrónicas.

Entre los cambios en la prensa escrita porteña de la segunda década del siglo $\mathrm{XX}$, dos modelos periodísticos, el de la prensa barata y el del periodismo "objetivo"4, han desplazado casi por completo al modelo de la prensa partidaria. El modelo de la"objetividad" periodística impuesto internacionalmente gracias al diario nuevoyorquino The New York Times, se instala como paradigma dominante a partir del rediseño que impulsa su editor, Alfred Ochs, en las postrimerías del siglo XIX ${ }^{5}$. Tal es la influencia de ambos modelos que incluso La Nación, un diario íntimamente asociado a las elites conservadoras de la Sociedad Rural y uno de los principales referentes de la prensa de partido en la Argentina, también se vuelca al nuevo contrato de lectura en 1914 alegando:

\begin{abstract}
Nuestras apreciaciones no interpretan el juicio, ni están sometidas al control de ningún comité: nuestras simpatías o nuestras resistencias, no nos obligan a asimilarnos, ni a empecinarnos con o contra las actuales o venideras fuerzas de opinión. [...] Fuimos un diario de partido, abierta y francamente, en días de grandes y famosas contiendas, pero dejamos de serlo, más cuando el genio de bien y virtud que inspiraba nuestra prédica dio por cerrado el ciclo de su acción (La Nación, 1914, apud Sidicaro, 1993, p. 46).
\end{abstract}

Durante las primeras dos décadas del siglo XX los diarios argentinos buscaron aproximarse a un público que gracias al voto popular había adquirido

4 Michael Schudson es quien mejor define la idea de "objetividad" en relación con su uso en el periodismo estadounidense. Para Schudson, objetividad remite a "una serie de afirmaciones sobre el mundo validadas consensualmente" (Schudson, 1978, p. 122; 144-159).

5 A partir de 1896 el desembarco de Alfred Ochs como editor de The New York Times, marca el cambio del paradigma de la "pennypress" de Hearst y Pulitzer al del periodismo "objetivo" (Schudson, 1978, p. 88-120). 
relevancia como actor político, un público que requería que la prensa lo mantuviese al corriente no sólo de temas de gobierno, sino de temas culturales, artísticos y científicos ${ }^{6}$.

Ya hacia 1913, los vespertinos de Buenos Aires comenzaron a mostrar en sus páginas una síntesis trabajosa de ambos modelos. Los títulos catástrofe, las ilustraciones, fotos y caricaturas, tomados del periodismo de Hearst y Pulitzer, sumados al afán de independencia, imparcialidad yla búsqueda dela"objetividad" del modelo impuesto por el New York Times nutrieron tanto a Crítica como a El Mundo y La Nación. La incorporación de estas técnicas, sin embargo, no habla de una adopción ciega de modelos extranjeros sino más bien de la utilización de estrategias periodísticas, publicitarias y comerciales para el desarrollo de un periodismo local todavía considerado como instrumento de acción política?.

Es en parte una combinación de ambos estilos periodísticos lo que define a Crítica durante las primeras tres décadas del Siglo XX.

La tensión entre ambos estilos se hace más que evidente durante la semana del 12 al 19 de agosto de 1933, la misma semana en la que el diario lanza la Revista Multicolor. En medio de una explosiva cobertura del asesinato del millonario Alberto de Álzaga, degollado en su departamento de la calle Charcas pocos días antes, el diario dedica abundante espacio editorial a temas literario-culturales como el arribo del escritor mexicano Alfonso Reyes a Buenos Aires ("Vengo a Descansar a Buenos Aires, Una Ciudad que se Contrae", Dice A. Reyes en Crítica del 12 de agosto de 1933, página 4), la visita del rector de la Universidad Central de España, Claudio de Albornoz ("Dr. Claudio de Albornoz. Llega a Nuestro País, el Espíritu de la Nueva Universidad Española”,en Crítica del 13 de agosto de 1933, página 14) además de ofrecer varias notas sobre los cambios políticos en Cuba, la declaración del estado de sitio en Irlanda, la crisis política entre Alemania y Austria, la huelga portuaria en Sevilla y los combates en Irak.

6 Gracias a la aprobación en 1912 de la Ley Sáenz Peña, que establece el sufragio obligatorio, universal y secreto, Hipólito Yrigoyen se convirtió en 1916 en el primer presidente Argentino electo por voto popular tras décadas de fraude electoral. En relación con este tema, ver: Botana (1985, p. 17). También ofrecen una perspectiva interesante la serie de documentos compilados por Ezequiel Gallo y Natalio Botana (2007, p. 599-634).

7 No está claro si Natalio Botana, fundador y editor de Crítica, tuvo contacto directo por el periodismo neoyorquino de William Randolph Hearst y Joseph Pulitzer de finales del Siglo XIX. Por el contrario, es bien sabido que en 1928 y 1929, tras de la muerte de su hijo adoptivo, Carlos Natalio, Botana viajó con su familia a Europa y Nueva York. Y allí, según Saítta (1998, p. 287), "fue capaz de observar las operaciones de algunos de los mayores diarios de Nueva York y adoptar [...] algunas prácticas que considera conveniente poner en práctica entre nosotros [en Crítica]". En la década de 1920, sin embargo, la era dorada de sensacionalismo y la prensa amarilla neoyorquina ya había quedado atrás, y el modelo de la objetividad, asociado al periodismo especializado e interpretativo, se convertía en el nuevo paradigma del diarismo de masas (Schudson, 1978, p. 122; 144-159). 
En las páginas de Crítica de este período, la titulación dramática y efectista, que casi siempre responde además al estilo que en el periodismo estadounidense se denomina de las $5 \mathrm{~W}$ (los títulos responden a las cinco preguntas básicas: quién, qué, dónde, cuándo y por qué, o who, what, where, when y why) se combina con textos que alternan entre la "pirámide invertida" (con encabezados y primeros párrafos que condensan los elementos más importantes de la noticia) y otros más decididamente narrativos.

Pero el objetivo central de Crítica fue el acceso de las masas populares a la lectura. El 6 de diciembre de 1924, al poner en marcha Biblioteca Crítica, una serie de libros baratos que acompañaron a diario Crítica, un editorial en el periódico refuerza esa idea:

\footnotetext{
El hombre que durante el día, agobiado por un rudo trabajo, desea entretener su descanso con la lectura, se encuentra ante un grave dilema: ¿qué puede leer? Carece de tiempo para buscar en una librería lo que más le conviene a su temperamento. Desconoce a los autores y a los libros mismos; no es erudito y quisiera a la vez un libro ameno e instructivo (Saitta, 1998, p. 74).
}

Además de las secciones de noticias, cargadas de historias de crimen e interés humano, los periódicos populares de esta época incorporaban también novelas por entregas, libros de bolsillo, y varios tipos de lecturas livianas y de entretenimiento. En ese contexto, Botana concibió la idea de un producto más sofisticado, de lectura a un nivel más elevado, que se animase a discutir obras literarias nuevas y clásicas, tanto de autores locales y como en traducción. Este producto consolidaría a Crítica - y a líder de opinión política - como una vitrina para las nuevas vanguardias literarias. En ese sentido, Crítica lideró en la Argentina la transformación del modelo de prensa política hacia un modelo de prensa moderna, basado en la información, un modelo que se puso en marcha casi cien años después que esa misma transformación ocurriese en los Estados Unidos. Esta brecha entre el norte y el sur del continente, tanto en la consolidación de dos sistemas democráticos como en el desarrollo de la prensa de masas, debe tenerse en cuenta a fin de comprender el modo en que Borges se relaciona con el periodismo y la literatura. 


\section{La historia universal de la infamia en el contexto de un la prensa del centavo}

Durante la semana del 12 al 19 de agosto de 1933, los dos vespertinos más importantes de Buenos Aires, El Mundo y Crítica, dedicaron una amplia cobertura al cruento asesinato del millonario Alberto de Álzaga. El cuerpo degollado de Álzaga, que había sido encontrado en el apartamento de la familia en la calle Charcas, una zona céntrica de Buenos Aires, fue tema central de la portada de Crítica durante toda la semana, y uno de los principales de la sección local de El Mundo. Los titulares a raíz de la investigación, las dudas sobre el testamento de Álzaga y el paradero desconocido del asesino, se adueñaron de las mayores cuotas del espacio editorial de portada de Crítica, espacio que apenas compartió el domingo 13 con un titular de deportes; el día 16 con la noticia de un ministro recién nombrado en la Argentina; el día 17 con un titular internacional sobre Bolivia; y el día 18 con otro artículo internacional sobre el colapso de las relaciones diplomáticas entre Alemania y Austria. Las notas, todas acompañadas por fotos del millonario muerto, sus familiares o los agentes de policía encargados de la investigación, fueron tapa, precisamente, en la semana en que por primera vez aparecería la Revista Multicolor de los Sábados ${ }^{8}$. En algunos de los textos sobre el asesinato de Álzaga, los reporteros de Crítica pedían incluso a sus lectores que contribuyeran directamente con la investigación mediante el envío de hipótesis propias, que se publicarían por separado. El crimen, uno los editores escribió: “... parece [sacado de] una novela de Conan Doyle" (Saitta, 1998, p. 208).

Se desprende de la lectura detallada del periódico durante esta semana, que la interacción entre diario y lectores funcionaba bajo un contrato de lectura que operaba a varios niveles diferentes. En tal sentido, la navegación de los matices que requiere una historia breve como "El Proveedor de iniquidades Monk Eastman" de Borges, no puede ser considerada como una desviación de las demandas habituales de lectura propuestas por Crítica. El cuento de Borges apareció el 19 de agosto, al mismo tiempo en que el asesinato de Alzaga era cubierto como una "novela de Conan Doyle".

8 "El Criminal está en Buenos Aires", 12 de agosto; "Ricardo Alzaga se presentó a la policía y fue puesto en Libertad", 13 de agosto; "La Pesquisa no marcha a pesar del optimismo", 14 de agosto; "Andan a tientas en la investigación y están desorientados", 15 de agosto; "Se busca al matador de Alzaga en Rosario", 16 de agosto; "El testamento tiene enmiendas", 17 de agosto; "Las alhajas de Alzaga darán una pista cierta" 18 de agosto; y "Más misterio: las diligencias de ayer", 19 de agosto. El Mundo, competidor directo de Crítica, también cubrió la saga del asesinato de Alzaga profusamente, pero debido a su contrato de lectura -la primera sección se enfoca sobre temas internacionales. Ambos periódicos fueron consultados en la Biblioteca Nacional, en Buenos Aires, Argentina. Hasta el cierre de este artículo, no existían microfilmes o copias digitales de los originales y algunas de las copias de Crítica aparecen deterioradas $y$, a veces, con secciones enteras faltantes. 
Pero ¿cómo podemos determinar si los lectores de la Revista Multicolor - y de los relatos de Borges, en particular- eran, de hecho, los mismos que se interesaban por el resto del diario? Tras ochenta y cinco años de la publicación original de estos relatos de Borges, sin un trabajo etnográfico en recepción, sólo podemos rastrear algunas marcas en el contexto de producción inicial del periódico, con la esperanza de que estas las señales nos ayuden a lograr un análisis más preciso.

En primer lugar, los anuncios de la Revista Multicolor durante sus dos primeras semanas aparecieron en la sección de Deportes de Crítica, el suplemento más "popular" del diario, una sección destinada a apelar al denominador común de los lectores porteños, escrito en prosa sencilla, con el más básico de los contenidos del periódico. El primer anuncio de la Revista Multicolor apareció el 11 de agosto, y describía los textos que publicaría el suplemento literario como "historias verdaderas", "historias reales", "artículos" o simplemente "historias". Curiosamente, sólo algunas de los escritos fueron clasificados en el anuncio como "cuentos ficticios". En el segundo anuncio de la revista, que apareció el 18de agosto, nuevamente en la portada de la sección de Deportes, sólo seis de las 17 historias eran descritas como cuentos o ficción. En dicho anuncio "El Proveedor de iniquidades Monk Eastman" no era mencionado ni como ficción ni como no ficción, sino simplemente como "Un capítulo de la Historia Universal de la Infamia". Estos anuncios fueron con seguridad supervisados por Borges y Petit de Murat (Saitta, 1998).

\section{"Monk Eastman": una lecturade aproximación contextual}

Crítica fue el terreno de pruebas perfecto para las primeras historias narrativas de Borges. Las piezas incluidas en la serie HUI pueden leerse como interactuando con el lector, ampliando el contexto de lectura de las noticias que aparecían en el periódico durante el curso de la semana. Green ya ha hablado de las similitudes estructurales entre las noticias publicadas en Crítica y los textos de HUI: las divisiones en las subsecciones, presentes tanto en las noticias del cuerpo central del diario como en los escritos del suplemento, o el uso sensacionalista de los subtítulos, son técnicas compartida por las secciones de noticias y la revista (Green, 2010).

Pero hay otras señales que proponen una nueva forma de lectura de los cuentos de Borges. "El Proveedor de iniquidades Monk Eastman", que apareció la misma semana del asesinato Alzaga, sigue de cerca el libro The Gangs of New York, de Herbert Asbury (1928). Balderston (2003), como se ha mencionado anteriormente, marca una serie de similitudes entre el original y el cuento de Borges, y ha propuesto que las alteraciones de Borges a la historia original 
fueron realizadas por el escritor para ampliarlos aspectos cinemáticos del relato. Sin embargo, al omitir el primer párrafo de la historia, el enmarque al comienzo de la historia, un fragmento que conecta al relato con el resto de las historias en el periódico, Balderston pierde de vista una pista esencial para la interpretación del cuento a un nuevo nivel.

Monk Eastman fue el líder de una de las bandas más violentas de Nueva York a fines del siglo 19 y comienzos del 20. Y, entre otras cosas, el cuento de Borges establece una comparación a gran escala entre la violencia de "Los de esta América"y la violencia de "Los de la otra" (ambos subtítulos fueron añadidos a la edición de 1935 del libro de la HUI, pero no aparecían en el original en Crítica). La comparación tiene, sin duda, sus raíces en un tema ya desarrollado por José Martí: la noción de "Nuestra América" como entidad cultural independiente y opuesta a los Estados Unidos (Calvi, 2011).

Por oposición a la vertiginosa, torpe, bárbara e inepta Nueva York, Borges retrata los bajos fondos de Buenos Aires de un modo estilizado y sobrio, honorable, elegante y limpio. Con fines de ilustrar la comparación, cito el pasaje en extenso:

Los de esta América Perfilados bien por un fondo de paredes celestes o de cielo alto, dos compadritos envainados en seria ropa negra bailan sobre zapatos de mujer un baile gravísimo, que es el de los cuchillos parejos, hasta que de una oreja salta un clavel porque el cuchillo ha entrado en un hombre, que cierra con su muerte horizontal el baile sin música. Resignado, el otro se acomoda el chambergo y consagra su vejez a la narración de ese duelo tan limpio. Ésa es la historia detallada y total de nuestro malevaje. La de los hombres de pelea de Nueva York es más vertiginosa y más torpe.

Los de la Otra La historia de las bandas de Nueva York (revelada en 1928 por Herbert Asbury en un decoroso volumen de cuatrocientas páginas en octavo) tiene la confusión y la crueldad de las cosmogonías bárbaras y mucho de su ineptitud gigantesca: sótanos de antiguas cervecerías habilitadas para conventillos de negros, una raquítica Nueva York de tres pisos, bandas de forajidos como los Ángeles del Pantano (Swamp Angels) que merodeaban entre laberintos de cloacas, bandas de forajidos como los Daybreak Boys (Muchachos del Alba) que reclutaban asesinos precoces de diez y once años, gigantes solitarios y descarados como los Galerudos Fieros (Plug Uglies) que procuraban la inverosímil risa del prójimo con un firme sombrero de copa lleno de lana y los vastos faldones de la camisa ondeados por el viento del arrabal, pero con un garrote en la diestra y un pistolón profundo; bandas de forajidos como los Conejos Muertos (Dead Rabbits) que entraban en batalla bajo la enseña de un conejo muerto en un palo; hombres como Johnny Dolan el Dandy, famoso 
por el rulo aceitado sobre la frente, por los bastones con cabeza de mono y por el fino aparatito de cobre que solía calzarse en el pulgar para vaciar los ojos del adversario; hombres como Kit Burns, capaz de decapitar de un solo mordisco una rata viva; hombres como Blind Danny Lyons, muchacho rubio de ojos muertos inmensos, rufián de tres rameras que circulaban con orgullo por él; filas de casas de farol colorado como las dirigidas por siete hermanas de New England, que destinaban las ganancias de Nochebuena a la caridad; reñideros de ratas famélicas y de perros, casas de juego chinas, mujeres como la repetida viuda Red Norah, amada y ostentada por todos los varones que dirigieron la banda de los Gophers; mujeres como Lizzie the Dove, que se enlutó cuando lo ejecutaron a Danny Lyons y murió degollada por Gentle Maggie, que le discutió la antigua pasión del hombre muerto y ciego; motines como el de una semana salvaje de 1863 , que incendiaron cien edificios y por poco se adueñan de la ciudad; combates callejeros en los que el hombre se perdía como en el mar porque lo pisoteaban hasta la muerte; ladrones y envenenadores de caballos como YoskeNigger -tejen esta caótica historia. Su héroe más famoso es Edward Delaney, alias William Delaney, alias Joseph Marvin, alias Joseph Morris, alias Monk Eastman, jefe de mil doscientos hombres (Borges, 1999) ${ }^{9}$.

Para los lectores de Crítica, y para los nuevos lectores de masas, que han venido siguiendo la zaga del cruento asesinato de Alfredo de Alzaga, leyendo página tras página historias de asesinatos y proxenetas, traficantes de drogas, los bajos fondos del más diverso origen y color, y las miserias más umbrosas de la sociedad argentina, los disturbios en Nueva York y sus edificios incendiados, sus proxenetas y ladrones no podían resultar totalmente inconcebibles. En tal sentido, "esta América" y los Estados Unidos han debido haber presentado similitudes evidentes ante el público lector de Crítica. Este es, proponemos aquí, uno de los intentos temáticos de Borges, quien para ello apela a un dispositivo retórico cercando a la contradicción: la antífrasis: referir lo contrario de lo que realmente se quiere de ci.

Las actividades de Monk Eastman durante las elecciones, pagas por los políticos de Tammany, debían resultar no sólo familiares para los lectores de Borges, sino casi moneda corriente, en especial como lectura de los periódicos argentinos de la "década infame". En especial dado que, a principios de los años 1930, Argentina ya había sufrido casi una década de fraude electoral, apoyado y practicado por los caudillos políticos locales, y tolerado en gran medida por la policía y las entidades oficiales ${ }^{10}$.

$9 \quad$ Itálicas agregadas por el autor para énfasis.

10 Ver Gallo y Botana (2007, p. 599-634); y una serie de documentos compilados por ellos. 
Es en este sentido que podemos comprender cómo Borges toma el tópico martiano de las dos Américas para resignificarlo. La historia de Borges sobre Nueva York, publicada en las páginas de un periódico popular de masas en argentina, se puede leer como una broma interna entre el escritor y el lector. Es una broma acerca de la política argentina que sólo cobra sentido en términos del contrato de lectura de Crítica. La comparación entre "esta América"y "la otra América" sólo puede ser entendida por aquellos que comprenden el contexto correctamente. La antífrasis separa a los que están "adentro" de los que se quedan "afuera", los buenos lectores y los malos. Cuando Borges intenta denotativamente establecer una separación entre Buenos Aires y los bajos fondos de Nueva York, está intentando mostrar a sus mejores lectores cuán cerca están realmente esos dos submundos. Al hacerlo, Borges integra a Buenos Aires al universo más amplio de la modernidad cosmopolita del Siglo XX, pareando a Buenos Aires con Nueva York. El efecto final de "El proveedor de iniquidades Monk Eastman" para los lectores de Crítica, sostengo aquí, ha de haber sido la alineación y la familiaridad entre Nueva York y Buenos Aires. Ambas ciudades quedan, por efecto de un artificio literario, situadas en paralelo, ocasial mismo nivel.

\section{Conclusiones: el embuste, los que de adentro y los de afuera}

Borges entendía HUI como un libro lúdico. Lo dejó claro en su prólogo a la edición de 1935. En ese texto describe a los buenos lectores como "cisnes aún más tenebrosos y singulares que los buenos autores", y a la lectura como " una actividad posterior a la de escribir: más resignada, más civil, más intelectual"(Borges, 1999). En las páginas de la Revista Multicolor, Borges imaginó a ese lector perfecto, un decodificador altamente capacitado para comprender interacciones y mensajes sutiles, pero también entrenado en la lectura de contexto.

"[Todas] las historias en el libro eran una especie de chistes ofalsificaciones", aseguró Borges (apud Burgin, 1970, p. 28) años después de la segunda edición de la HUI durante una conversación con Richard Burgin. "Pero ahora no pienso demasiado en ese libro, me hacía mucha gracia cuando lo escribí, pero no puedo recordar quiénes eran los personajes" (Burgin, 1970, p. 28). La falsa modestia de Borges, sin embargo, no debe distraernos porque las historias en el libro, publicadojustamente en un periódico popular de masas, sítuvieron un propósito.

En su excelente Froth and Scum, Andie Tucher discute el papel de la broma y el embuste ("the hoax") como práctica periodística común en la prensa del penique durante la democracia jacksoniana estadounidense. Durante ese período, historias falsas o apócrifas eran publicadas lado a lado con las noticias "reales", para que los lectores decidiesen acerca de su autenticidad o su 
falsedad absoluta. "La resolución de un engaño y el trabajo intelectual que esto implicaba, en fin, les demandaba a todos los ciudadanos el trabajo democrático de evaluar", explica Tucher.

\begin{abstract}
Se ofrecía asía los ciudadanos la experiencia democrática de participar en la vida pública [...] En un sentido importante, sin embargo, un embuste se parece a una mentira. Hay víctimas. No se las daña de modo directo; no pierden dinero. A menudo ni siquiera saben que son víctimas. Sin embargo, sin ellos no existiría el embuste. Ellos son los que no comprendieron, los que quedaron afuera (Tucher, 1994, p. 57) ${ }^{11}$.
\end{abstract}

Cuando supe hace unos 25 años que Borges había escrito sus primeros relatos en prosa en el diario Crítica, fui directamente a mi abuelo. Un voraz lector de periódicos, devoto de El Mundo, él también estaba familiarizado con Crítica. Hijo de inmigrantes españoles, mi abuelo había hecho carrera en el rubro de venta al por menor y en la década de 1930 había llegado a la cima de su carrera trabajando en la tienda Harrods Gath\&Chavez, en pleno centro de Buenos Aires. Cuando le pregunté acerca de Borges, y si lo había leído, su respuesta fue inequívoca: "Era un cómico. Escribía en la página de chistes de Crítica". En parte, creo que es así como la primera obra narrativa de Borges fue percibida por los lectores que, como mi abuelo, vivieron en carne propia la Década Infame, sangrienta y corrupta. Lectores que fueron testigos mediáticos de un asesinato en la Cámara Alta de la Nación, lectores que también sufrieron en carne propia la intimidación de los punteros políticos locales en día de elecciones. En esos años, cuando el diarismo argentino comienza a gravitar hacia el modelo de la prensa de masas, más democrático, más inclusivo, la HUI de Borges debía ser leída por los lectores de Crítica como un embuste, como una broma. Y, al igual que los embustes de la prensa jacksoniana en los Estados Unidos dirigidos a los nuevos agentes del sistema democrático cien años antes, la HUl sirvió también a un propósito superior: estimular el diálogo colectivo en los cafés y los pasillos, los autobuses y las plazas, en el contexto de una nación democrática naciente, joven, urbana y vibrante.

11 Traducción del autor. 


\section{Referencias}

ALAZRAKI, Jaime. Oxymoronic Structure in Borges Essays. Books Abroad 45, p. 42127, Summer 1971.

ALONSO, Amado. Borges narrador. Sur 14, Valdivia - Chile, p. 105-15, 1935.

BALDERSTON, Daniel. Out of Context: Historical Reference and the Representation of Reality in Borges. Duke University Press, 1993.

Borges and the Gangs of New York. Variaciones Borges, v. 16, p. 27-33, 2003.

BORGES, Jorge Luis. Ensayo Autobiogáfico. La Opinión, Sep., v. 17, p. 16, 1974.

Collected Fictions. New York: Penguin Press, 1999.

Obras Completas, Tomo 1, Buenos Aires: EMECE, 1999.

BOTANA, Natalio e outros. El Marco Histórico Institucional: Leyes Electorales, Alternancia y Competencia entre Partidos. La Argentina electoral, Buenos Aires, 1985.

BURGIN, Richard. Conversations with Jorge Luis Borges. New York: Avon Books, 1970.

CALVI, P.The Parrot and the Cannon. Journalism, Literature and Politics in the Formation of Latin American Identities, p. 171-184, Columbia University: Academic Commons, 2011. Disponible en: <http://academiccommons.columbia.edu/ catalog/ac\%3A174251>. Acceso en: 20 ago. 2016.

EUJANIÁN, Alejandro. Historia de Revistas Argentinas 1900-1950. La Conquista del Público. Buenos Aires: Asociación Argentina de Editores de Revistas, 1999.

GALLO, E.; BOTANA, Natalio. De la república posible a la república verdadera. Buenos Aires: Emecé, 2007.

GREEN, Raquel Atena. Borges y Revista Multicolor de los Sábados. Confabulados en una escritura de la infamia. New York: Peter Lang, 2010.

LA NACIÓN. 3 de abril de 1914. In: Sidicaro, Ricardo. La política mirada desde arriba. Las ideas del diario La Nación, 1909-1989. Buenos Aires: Sudamericana, 1993.

LEWIS, Annick. Instrucciones para buscar a Borges en la Revista Multicolor. Variaciones Borges, Pittsburgh, v. 5, p. 246-264, 1998.

MARTÍ, José. Crónicas. Disponible en: <http://www.biblioises.com.ar/ Contenido/800/863/Jose\%20Marti\%20-\%20Cronicas.pdf>. Acceso en: 19. ago. 2016.

MOLLOY, Sylvia. Signs of Borges. Durham: Duke University Press, 1994. 
Las letras de Borges y otros ensayos. Rosario: Beatriz Viterbo, 2000.

RIVERA, Jorge B. Los juegos de un tímido: Borges en el suplemento de Crítica. Crisis, Mayo-Junio 1976, p. 20-24. Reeditado por Ford, Aníbal, Jorge Rivera, Eduardo Romano. Medios de comunicación y cultura popular. Buenos Aires: Legas, 1985.

SAÍTTA, Sylvia. Regueros de tinta. El diario Crítica en la década de 1920. Buenos Aires: Sudamericana, 1998.

De este lado de la verja: Jorge Luis Borges y los usos del periodismo moderno. Variaciones Borges, Pittsburgh, v. 9, p. 74-83, 2000.

SCHUDSON, Michael. Discovering the News. A Social History of American Newspapers, 1978.

TORRES, Jose Luis. La Década Infame. Buenos Aires: Patria, 1945.

TALICE, Roberto. $\mathbf{1 0 0 . 0 0 0}$ ejemplares por hora. Memorias de un redactor de Crítica, el diario de Botana. Buenos Aires: Ediciones del Corregidor, 1989.

TUCHER, Andie. Froth and Scum. Truth. Beauty. Goodness. And the Ax Murder in America's First Mass Medium. Chapel Hill:The University of North Carolina Press, 1994.

VASQUEZ, Marlene. Martí y América. Permanencia del Diálogo. abrapalabra n. 36, 2004, Santa Clara, Cuba, DR. Disponible en: <http://biblio3.url.edu.gt/ Libros/2011/marti amer.pdf>. Acceso en: 15 ago. 2016.

WELLS, Sarah Ann. Late Modernism, Pulp History: Jorge Luis Borges A Universal History of Infamy, 1935. MODERNISM / modernity, Baltimore, v. 18, n. 2, p. 425-441, 2011.

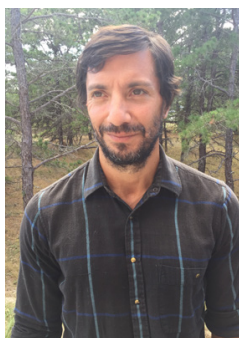

Recebido em: 31/8/2016

Aceito em: 31/8/2016

Endereço do autor:

Pablo Calvi <pablo.calvi@stonybrook.edu>

Stony Brook University's School of Journalism

11794 - Stony Brook - New York - EUA 